\title{
Nonlinear Thomson scattering: A tutorial ${ }^{\mathrm{a})}$
}

\author{
Y. Y. Lau, ${ }^{\text {b) }}$ Fei He, Donald P. Umstadter, and Richard Kowalczyk \\ Department of Nuclear Engineering and Radiological Sciences, University of Michigan, Ann Arbor, \\ Michigan 48109-2104
}

(Received 13 November 2002; accepted 10 February 2003)

\begin{abstract}
Recent advances in table-top, ultrahigh intensity lasers have led to significant renewed interest in the classic problem of Thomson scattering. An important current application of these scattering processes is the generation of ultrashort-pulse-duration $\mathrm{x}$ rays. In this tutorial, the classical theory of nonlinear Thomson scattering of an electron in an intense laser field is presented. It is found that the orbit, and therefore its nonlinear scattering spectra, depends on the amplitude and on the phase at which the electron sees the laser electric field. Novel, simple asymptotic expansions are obtained for the spectrum of radiation that is backscattered from a laser by a counter-propagating (or co-propagating) electron. The solutions are presented in such a way that they explicitly show-at least in the single particle regime- the relative merit of using an intense laser and of an energetic electron beam in x-ray production. The close analogy with free electron laser/synchrotron source is indicated. (C) 2003 American Institute of Physics. [DOI: 10.1063/1.1565115]
\end{abstract}

\section{INTRODUCTION}

The widespread availability and the continuous development of ultra-high intensity lasers have created the exciting field of "High Field Science." Many regimes that were deemed out of reach just a decade ago suddenly became closer to reality. Important applications include table-top electron and proton accelerators, advanced x-ray sources, medical isotope production, ultrafast imaging, laser surgery, and materials treatment. ${ }^{1-4}$

In this tutorial, we focus only on one aspect, namely, the generation of $x$ rays by Thomson scattering of an intense laser by electrons. ${ }^{5-32}$ The energetic electrons may be generated separately by a conventional high energy accelerator, or by another intense laser pulse via one of the several advanced acceleration mechanisms. ${ }^{33}$ If a laser-based accelerator were used in conjunction with a synchronized scattering laser pulse, then an all-optical $\mathrm{x}$-ray source would be possible. ${ }^{5,19,32}$ Applications of Thomson/Compton x-ray sources include dynamical studies and imaging of solid, molecular, and biological systems. ${ }^{34-37}$

In Thomson scattering, an electron that is initially at rest may acquire relativistic velocities in the fields of highintensity light and through this relativistic motion the electron may emit radiation at high harmonics of the light frequency. If the electron already possesses a relativistic energy before it encounters the high-intensity laser, there is an additional Doppler-shift of the scattered light. By Thomson scattering, we mean that the photon energy $(h v)$ of the scattered radiation is much less than the electron energy $\left(m c^{2}\right)$, or $h v \ll m c^{2}$, while for Compton scattering, this condition is not met. As an example of Thomson scattering, electrons with only $100 \mathrm{MeV}$ energy can upshift a $1 \mathrm{eV}$ photon to an energy of $50 \mathrm{keV}$, which is of interest not only as a probe with

\footnotetext{
a)Paper GI2 1, Bull. Am. Phys. Soc. 47, 136 (2002).

${ }^{b)}$ Invited speaker.
}

atomic-scale spatial resolution, but also as a medical diagnostic. Several proposals have been made to build a "gamma-gamma" collider for high-energy physics experiments, in which $200 \mathrm{GeV}$ gamma rays are generated by Compton scattering of $1 \mathrm{eV}$ photons from $250 \mathrm{GeV}$ energy electron beams. Colliding such energetic photons to create particles through the inverse process has advantages over direct particle collisions because of reduced beamstrahlung and disruption. In all cases of scattering, the electromagnetic wave acts as an undulator/wiggler, replacing the alternating static magnetic field used in conventional synchrotrons or free electron lasers (FEL) ${ }^{34}$ The field strength in the former case can be orders-of-magnitude higher, and the length, orders-of-magnitude shorter, than in the latter case. The results of numerous experiments, ${ }^{37-42}$ theories, ${ }^{43-46}$ and reviews ${ }^{4,31}$ related to these topics have been published. Parallel efforts in the FEL/synchrotron community are documented in Refs. 47-83. Nonlinear Thomson and Compton scattering was also studied in Refs. 84-90.

While an $\mathrm{x}$-ray source based on laser scattering 5,6,10,28,32,45 and one based on the conventional synchrotron/FEL mechanism ${ }^{34,66,92}$ differ in the characteristics of undulator/wiggler used, their physical and mathematical descriptions are strikingly similar. In fact, the normalized laser electric field, $a$, and the normalized wiggler parameter, $K$, are almost interchangeable in the description of Thomson scattering for head-on collisions between a relativistic electron against a laser. Here we present the solution of the backscatter spectrum for arbitrary laser intensity and arbitrary electron energy (including zero), and the simple asymptotic expressions recently obtained for the various regimes. ${ }^{90}$ The solutions are presented in such a way that they explicitly show the relative merit of using an intense laser and of an energetic electron beam in x-ray production in the single particle regime. We also indicate the origin of a number of misconceptions that appear in the literature in the ultraintense laser community [cf. paragraph following Eq. (11)]. 
The laser electric field is measured by the dimensionless parameter, $a=e E_{0} / m \omega_{0} c$, where $E_{0}$ is the electric field amplitude, $\omega_{0}$ is the laser frequency, and $m$ is the electron rest mass. For a linearly polarized laser field,

$$
\begin{aligned}
a & =0.85\left(\frac{\lambda}{1 \mu \mathrm{m}}\right)\left(\frac{I}{10^{18} \mathrm{~W} / \mathrm{cm}^{2}}\right)^{1 / 2} \\
& =0.31\left(\frac{\lambda}{1 \mu \mathrm{m}}\right)\left(\frac{E_{0}}{100 \mathrm{~V} / \AA}\right)=0.22\left(\frac{\lambda}{r_{s}}\right)\left(\frac{P}{1 \mathrm{GW}}\right)^{1 / 2}
\end{aligned}
$$

in terms of the laser wavelength $\lambda$, laser intensity $I$, laser power $P$, and the rms radius $r_{s}$ of the laser spot size (assuming a Gaussian profile). Physically, $a$ measures the transverse momentum, in units of $m c$, imparted by the oscillating laser field upon an electron.

For lasers with low power intensities $(a \ll 1)$, an electron that is initially at rest undergoes a small amplitude, transverse oscillation at the laser frequency $\omega_{0}$. The Thomson scattering spectrum consists of a single frequency $\omega_{0}$ in all directions and the radiation pattern is the same as that from a dipole antenna. As $a$ is increased to a value of a few tens of percent, the electron's oscillation frequency begins to deviate from the laser frequency. As the laser amplitude increases to $a=O(1)$, the Lorentz force associated with the laser's magnetic field becomes significant, and the electron acquires an oscillation along $\mathbf{k}$, the direction of laser propagation, in addition to the transverse oscillation. The electron also acquires an average drift velocity along k. For $a \gg 1$, the axial excursion of electron oscillation greatly exceeds the transverse excursion, and the electron orbital period is much greater than the laser optical period. At present, the achievable values of $a$ are in the single digits. Values of $a$ in the tens or even hundreds are being actively pursued in the "high field science" community. $4,28,45$

In Sec. II, we derive the general formula for the radiation spectrum, and point out some subtlety in its evaluation. In Sec. III, we describe the electron orbit subject to an intense laser field, displaying the potential importance of the phase. In Sec. IV, we consider the backscatter spectra in detail. The asymptotic scaling suggests the optimal combination of the laser and the electron beam for the brightest x-ray source. We conclude in Sec. V with a discussion of the various issues.

\section{RADIATION SPECTRA}

Once the orbit of a charged particle is known, the radiation spectrum may be derived using relativistic mechanisms, as done in Jackson. ${ }^{91}$ Alternatively, one may use a nonrelativistic treatment by starting with the first two of the Maxwell's equations, $i \mathbf{k} \times \mathbf{E}_{\mathrm{F}}=i(\omega / c) \mathbf{B}_{\mathrm{F}}, i \mathbf{k} \times \mathbf{B}_{\mathrm{F}}=4 \pi \mathbf{J}_{\mathrm{F}} / c$ $-i(\omega / c) \mathbf{E}_{\mathrm{F}}$, written in terms of the Fourier transforms (subscript $\mathrm{F}$ ) of the electric field $\mathbf{E}(\mathbf{r}, t)$, magnetic field $\mathbf{B}(\mathbf{r}, t)$, and current density $\mathbf{J}(\mathbf{r}, t)$, defined as

$$
\begin{aligned}
& {\left[\mathbf{E}_{\mathrm{F}}(\mathbf{k}, \omega), \mathbf{B}_{\mathrm{F}}(\mathbf{k}, \omega), \mathbf{J}_{\mathrm{F}}(\mathbf{k}, \omega)\right]} \\
& \quad=\int d t d^{3} \mathbf{r}[\mathbf{E}(\mathbf{r}, t), \mathbf{B}(\mathbf{r}, t), \mathbf{J}(\mathbf{r}, t)] e^{i \omega t-i \mathbf{k} \cdot \mathbf{r}} .
\end{aligned}
$$

Upon eliminating $\mathbf{B}_{\mathrm{F}}$ from these two Maxwell equations, one may find that $\mathbf{E}_{\mathrm{F}}=\mathbf{M} \mathbf{J}_{\mathrm{F}}$ where $\mathbf{M}$ is a matrix which depends only on $\mathbf{k}, \omega{ }^{34,92}$ The total work done, $W$, in ergs, performed by the current $\mathbf{J}(\mathbf{r}, t)$ on the electric field $\mathbf{E}(\mathbf{r}, t)$ is given by

$$
\begin{aligned}
W & =\int d t d^{3} \mathbf{r} \mathbf{J}(\mathbf{r}, t) \cdot \mathbf{E}(\mathbf{r}, t) \\
& =\frac{1}{(2 \pi)^{4}} \int d \omega d^{3} \mathbf{k} \mathbf{J}_{\mathrm{F}}(\mathbf{k}, \omega) \cdot \mathbf{E}_{\mathrm{F}}^{*}(\mathbf{k}, \omega),
\end{aligned}
$$

where we have used the Parseval's relation in writing the last expression in which the asterisk denotes the complex conjugate. Upon using the matrix $\mathbf{M}$ and the $\mathbf{k}$-space differential volume $d^{3} \mathbf{k}=k^{2} d k d \Omega$ which is expressed in terms of the solid angle $(\Omega)$ in the direction of the unit vector $\mathbf{n}=c \mathbf{k} / \omega$, Freund $^{92}$ in effect obtains the general formula for Eq. (2),

$$
\begin{aligned}
W & \equiv \int d \omega d \Omega \frac{d^{2} W}{d \omega d \Omega} \\
& =\int d \omega d \Omega \frac{\omega^{2}}{4 \pi^{2} c^{3}}\left[\left|\mathbf{J}_{\mathrm{F}}(\mathbf{k}, \omega)\right|^{2}-\left|\mathbf{n} \cdot \mathbf{J}_{\mathrm{F}}(\mathbf{k}, \omega)\right|^{2}\right] .
\end{aligned}
$$

Note that the square bracket in Eq. (3) is simply $\left|\mathbf{n} \times\left[\mathbf{n} \times \mathbf{J}_{\mathrm{F}}(\mathbf{k}, \omega)\right]\right|^{2}$.

An electron with displacement $\mathbf{r}(t)$ and velocity $\mathbf{v}(t)$ carries a current density $\mathbf{J}(\mathbf{r}, t)=e \mathbf{v}(t) \delta[\mathbf{r}-\mathbf{r}(t)]$, whose Fourier transform $\mathbf{J}_{F}(\mathbf{k}, \omega)$ may easily be obtained from Eq. (1). We immediately obtain from Eq. (3) the Jackson formula under the far-field approximation, ${ }^{91}$

$$
\begin{aligned}
& \frac{d^{2} W}{d \Omega d \omega}=\frac{e^{2} \omega^{2}}{4 \pi^{2} c}|\mathbf{n} \times[\mathbf{n} \times \mathbf{F}(\omega)]|^{2}, \\
& \mathbf{F}(\omega)=\int_{-\infty}^{\infty} d t \boldsymbol{\beta}(t) e^{i \omega[t-\mathbf{n} \cdot \mathbf{r}(t) / c]} .
\end{aligned}
$$

Equation (4) gives the energy radiated by the electron in the direction of the unit vector $\mathbf{n}$, per unit solid angle $\Omega$, per unit frequency $\omega$. Radiation damping is ignored throughout, and all calculations are in the lab frame.

Let us consider the simplest case where the electron orbit is strictly a periodic function of time with period $T$, and over one period, the electron undergoes a net displacement $\mathbf{r}_{0}$. Thus, we have for all integers $m$ (positive, negative or zero),

$$
\boldsymbol{\beta}(t+m T)=\boldsymbol{\beta}(t), \quad \mathbf{r}(t+m T)=m \mathbf{r}_{0}+\mathbf{r}(t) .
$$

Equation (5) may then be written as

$$
\begin{aligned}
\mathbf{F}(\omega) & =\sum_{m=-\infty}^{\infty} \int_{m T}^{(m+1) T} d t \boldsymbol{\beta}(t) e^{i \omega[t-\mathbf{n} \cdot \mathbf{r}(t) / c]} \\
& =\sum_{m=-\infty}^{\infty} \mathbf{f}(\omega) e^{i m \omega\left[T-\mathbf{n} \cdot \mathbf{r}_{0} / c\right]}
\end{aligned}
$$

where

$$
\mathbf{f}(\omega)=\int_{0}^{T} d t \boldsymbol{\beta}(t) e^{i \omega[t-\mathbf{n} \cdot \mathbf{r}(t) / c]}
$$


and we have used Eq. (6). Upon using $\Sigma_{m} e^{i m x}=\Sigma_{m} 2 \pi \delta(x$ $-2 m \pi$ ) in the last infinite sum in Eq. (7) and the property of the Dirac delta function, $\delta(a x)=(1 / a) \delta(x)$, we obtain from Eqs. (7) and (8) the following expression for the spectrum:

$$
\begin{aligned}
& \mathbf{F}(\omega)=\sum_{m=-\infty}^{\infty} \mathbf{F}_{m} \delta\left(\omega-m \omega_{1}\right), \\
& \omega_{1}=\frac{2 \pi}{T-\mathbf{n} \cdot \mathbf{r}_{0} / c}, \\
& \mathbf{F}_{m}=\frac{\omega_{1}}{2 \pi} \int_{0}^{T} d t \boldsymbol{\beta}(t) e^{i m \omega_{1}[t-\mathbf{n} \cdot \mathbf{r}(t) / c]} .
\end{aligned}
$$

Note from Eq. (9) that the radiation spectrum is discrete, for strictly periodic motion of the electron. The base frequency of this spectrum, $\omega_{1}$, depends on the orbital period $(T)$ of the electron, on the electron's net displacement $\left(\mathbf{r}_{0}\right)$ in one such period, and on the direction (n) in which the radiation is observed. Thus, the radiation spectrum is in general not at the harmonic frequency of the laser (nor at the harmonics of the electron orbital frequency, $2 \pi / T)$. It would thus be wrong to simply insert $\omega=n \omega_{0}$ in Eq. (8) and to replace the electron's orbital period $T$ there by the laser's optical period $2 \pi / \omega_{0}$ and consider the resultant value of that integral to give the spectral amplitude of the radiation at the $n$th harmonic of the laser frequency. Erroneous conclusions regarding generation of high laser harmonic have appeared in the literature based on such an intuitive (but incorrect) substitution.

The power, $p_{m}$ (in erg/s), radiated at the harmonic frequency $\omega=m \omega_{1}$ per unit solid angle in the direction of the unit vector $\mathbf{n}$ is then given by [cf. Eqs. (4) and (9)]

$$
p_{m}=\frac{e^{2} m^{2} \omega_{1}^{2}}{4 \pi^{2} c}\left|\mathbf{n} \times \mathbf{F}_{m}\right|^{2},
$$

where the dimensionless spectral amplitude $\mathbf{F}_{m}$ is given in Eq. (11). It is easy to show from Eqs. (6) and (10) that the integrand in Eq. (11) is a periodic function of $t$ of period $T$. Integral of this type is readily evaluated by the Romberg method. ${ }^{93}$ The electron orbit is considered next.

The formulas displayed in Secs. II and IV are in dimensional form. The formulas displayed in Sec. III have been normalized, as indicated.

\section{ELECTRON ORBITS}

The electron orbit subject to a linearly polarized electromagnetic wave propagating in the $+z$ direction (Fig. 1) is governed by the relativistic Lorentz equation

$$
\frac{d}{d t}(\gamma \boldsymbol{\beta})=(\mathbf{x}+\boldsymbol{\beta} \times \mathbf{y}) a \cos (t-z),
$$

where, as in this section, we normalize time by $1 / \omega_{0}$, velocity by $c$, and distance by $c / \omega_{0}$. In Eq. (13), $a$ $=e E_{0} / m \omega_{0} c$ is the dimensionless parameter measuring the electric field strength, $\gamma=\left(1-\beta_{x}^{2}-\beta_{y}^{2}-\beta_{z}^{2}\right)^{-1 / 2}$ is the relativistic mass factor, and $\boldsymbol{\beta}=\left(\beta_{x}, \beta_{y}, \beta_{z}\right)$ is the electron ve-

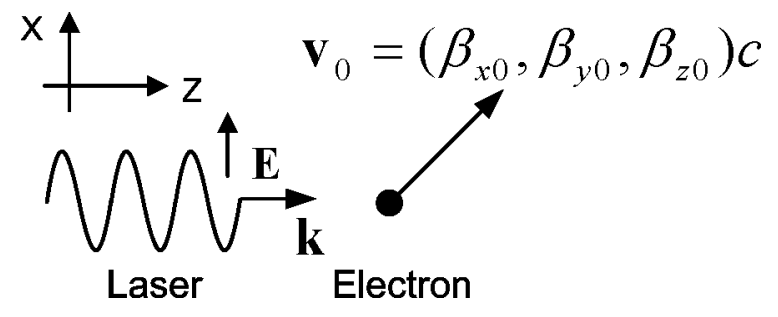

FIG. 1. The geometry. The laser propagates in the $+\mathbf{z}$ direction, a relativistic electron colliding head-on with the laser has $\beta_{x 0}=0, \beta_{y 0}=0, \beta_{z 0} \rightarrow$ -1 , and the laser backscatter is in the $\mathbf{n}=-\mathbf{z}$ direction.

locity (in units of $c$ ). The electron orbit, subject to the following general initial conditions at time $t=0$,

$$
\begin{aligned}
& x=0, \quad y=0, \quad z=z_{\text {in }}, \\
& \beta_{x}=\beta_{x 0}, \quad \beta_{y}=\beta_{y 0}, \quad \beta_{x}=\beta_{z 0},
\end{aligned}
$$

has a closed form solution when it is expressed parametrically: $t=t(\theta), \mathbf{r}=\mathbf{r}(\theta), \boldsymbol{\beta}=\boldsymbol{\beta}(\theta)$, where $\theta$ is the phase of the wave experienced by the electron and is defined by $^{7-13,26-28,30}$

$$
\theta=t-z
$$

Note that $\boldsymbol{\beta}_{0}=\left(\beta_{x 0}, \beta_{y 0}, \beta_{z 0}\right)$ is the unperturbed velocity of the electron ( $a=0$ limit; see Fig. 1$)$ and that the initial phase that the electron sees is $\theta_{\text {in }}=-z_{\text {in }}$ according to Eqs. (14a) and (15). This phase, included in Refs. 13 and 30, could be important in the ionization of the gas by an intense laser. ${ }^{94}$

For the special case $\beta_{x 0}=0, \beta_{y 0}=0$, one finds $\beta_{y}=0$, and the orbital equation yields the following closed form solution:

$$
\gamma=\gamma_{0}+\frac{a^{2}\left(\sin \theta-\sin \theta_{\text {in }}\right)^{2}}{2 \gamma_{0}\left(1-\beta_{z 0}\right)},
$$

$\gamma \beta_{z}=\gamma-\gamma_{0}\left(1-\beta_{z 0}\right)$,

$\gamma \beta_{x}=\frac{1}{\gamma_{0}\left(1-\beta_{z 0}\right)} a\left(\sin \theta-\sin \theta_{\text {in }}\right)$,

$x=\frac{a\left[\left(\cos \theta_{\text {in }}-\cos \theta\right)-\left(\theta-\theta_{\text {in }}\right) \sin \theta_{\text {in }}\right]}{\gamma_{0}\left(1-\beta_{z 0}\right)}$,

$t=\frac{\left(\theta-\theta_{\text {in }}\right)}{1-\beta_{z 0}}\left[1+\frac{a^{2}\left(1+\beta_{z 0}\right)}{2}\left(\frac{1}{2}+\sin ^{2} \theta_{\text {in }}\right)\right]+\frac{a^{2}\left(1+\beta_{z 0}\right)}{2\left(1-\beta_{z 0}\right)}$

$$
\times\left[-\frac{\sin 2 \theta}{4}+2 \cos \theta \sin \theta_{\text {in }}-\frac{3 \sin 2 \theta_{\text {in }}}{4}\right] .
$$

In Eqs. (16) $-(20), \gamma_{0}=\left(1-\beta_{z 0}^{2}\right)^{-1 / 2}$ and $\beta_{z 0}$ may either be negative (electron counter-propagates against the laser), or zero (electron initially at rest), or positive (electron co-propagates with the laser; see Fig. 1). Note that the velocity components $\beta_{x}$ and $\beta_{z}$ are given as explicit functions of $\theta$ according to Eqs. (17) and (18) upon using Eq. (16). They are periodic functions of $\theta$ of period $2 \pi$. The period, $T$, 
of this periodic ("figure- 8 ") motion is thus equal to the increase in $t$ as $\theta$ increases by $2 \pi$. Thus, we obtain from Eq. (20),

$$
T=\frac{2 \pi}{1-\beta_{z 0}}\left[1+\frac{a^{2}\left(1+\beta_{z 0}\right)}{2}\left(\frac{1}{2}+\sin ^{2} \theta_{\text {in }}\right)\right] .
$$

The parametric solution for the $z$ coordinates of the electron orbit is given by $z=t-\theta$ in which $t$ is given by Eq. (20). Over one orbital period, $T$, the electron undergoes a net displacement $\mathbf{r}_{0}=\left(x_{0}, 0, z_{0}\right)$ where $x_{0}$ is given by the increase in Eq. (19) as $\theta$ increases by $2 \pi$, and $z_{0}$ is simply $T-2 \pi$,

$z_{0}=T-2 \pi=\frac{2 \pi}{1-\beta_{z 0}}\left[\beta_{z 0}+\frac{a^{2}\left(1+\beta_{z 0}\right)}{2}\left(\frac{1}{2}+\sin ^{2} \theta_{\text {in }}\right)\right]$,

$x_{0}=\frac{-2 \pi a \sin \theta_{\text {in }}}{\gamma_{0}\left(1-\beta_{z 0}\right)}$.

Note that the electron trajectory depends on $a, \beta_{z 0}$, and $\theta_{\text {in }}$ in a rather complicated manner. Accordingly, the fundamental frequency $\omega_{1}$ of the radiation spectrum depends on these three quantities. ${ }^{30}$ In Sec. IV, we present the spectral solution for backscattered radiation $(\mathbf{n}=-\mathbf{z}$; see Fig. 1), in which case the dependence on electron beam and on the laser becomes decoupled.

Before we leave this section, we record a useful formula relating the change of $t$ with respect to $\theta$,

$$
\frac{d \theta}{d t}=1-\beta_{z}=\frac{\gamma_{0}\left(1-\beta_{z 0}\right)}{\gamma},
$$

which may be verified from Eqs. (15) and (17). Equation (24) is also valid for arbitrary values of $\beta_{x 0}, \beta_{y 0}, \beta_{z 0}$, and $\theta_{\text {in }}$, in which case $\gamma_{0}$ is the electron's relativistic mass factor in the absence of the laser.

\section{SPECTRAL DEPENDENCE ON ELECTRON BEAM AND LASER}

The orbital periodicity, $T$, together with the net orbital displacement, $\mathbf{r}_{0}$, as given by Eqs. (21)-(23) in normalized form, determine the fundamental frequency $\omega_{1}$ of the radiation spectrum when observed in the direction $\mathbf{n}$ [cf. Eq. (10), which is in the dimensional form]. The radiated power $\left(p_{m}\right)$ at the discrete frequencies $\left(\omega=m \omega_{1}\right)$ may then be obtained from Eq. (12) in which $\mathbf{F}_{m}$ may be computed from the orbital equations using the transformation from $t$ to $\theta$ in Eq. (24). Thus, the spectrum depends on $\omega_{0}, a, \theta_{\text {in }}, \mathbf{n}$, and $\beta_{0} .{ }^{30}$ Equations (21)-(23), together with Eq. (10), show that for $a^{2} \gg 1$, the dependence of $\omega_{1}$ on the phase is strong and $\omega_{1}$ $\sim 1 / a^{2}$. This dependence on the phase is weak for $a^{2} \ll 1$, in which case $\omega_{1} / \omega_{0}$ approaches unity (see Fig. 2 of Ref. 30). The radiation spectrum, in general, is not at integer harmonics of the laser frequency $\omega_{0}$.

The radiation spectrum observed exactly in the forward direction of the laser $(\mathbf{n}=\mathbf{z})$ always has only one discrete frequency, $\omega=\omega_{1}=\omega_{0}$. This is easily shown from Eq. (10) (which is in dimensional form), upon using the first equality of Eq. (22) (which is in dimensionless form). This statement is true regardless of the velocity of the electron or the laser intensity, and may easily be deduced from Eq. (11) for this case. However, for an energetic electron beam that is almost co-propagating with the laser, such as that produced by the laser itself, high harmonics at the laser frequency may be observed in the direction just slightly off the laser direction. This was used to explain the University of Michigan experiments on Thomson scattering. ${ }^{95}$

In the backscattering direction of the laser $(\mathbf{n}=-\mathbf{z}$, see Fig. 1), if one sets $\theta_{\text {in }}=0$, one obtains the following expressions for $\omega_{1}$ and $p_{m}$, the backscatter power per unit solid angle at $\omega=m \omega_{1}$ [cf. Eq. (12)], in dimensional form:

$$
\begin{aligned}
& \frac{\omega_{1}}{\omega_{0}}=\left(\frac{2}{2+a^{2}}\right)\left(\frac{1-\beta_{z 0}}{1+\beta_{z 0}}\right)=\left(\frac{2}{2+a^{2}}\right) \gamma_{0}^{2}\left(1-\beta_{z 0}\right)^{2}, \\
& p_{m}=\frac{A}{\gamma_{0}^{2}\left(1-\beta_{z 0}\right)^{2}}\left(\frac{\omega_{1}}{\omega_{0}}\right)^{4} s_{m},
\end{aligned}
$$

where $A=e^{2} \omega_{0}^{2} / 4 \pi^{2} c=0.69[\lambda /(1 \mu \mathrm{m})]^{-2} \mathrm{erg} / \mathrm{s}, s_{m}=0$ for $m=0, \pm 2, \pm 4, \ldots$, and for $m= \pm 1, \pm 3, \pm 5, \ldots$,

$$
\begin{aligned}
s_{m}= & (a \pi)^{2} m^{2}\left[J_{(m-1) / 2}(m \xi)-J_{(m+1) / 2}(m \xi)\right]^{2} \\
& (m=\text { odd }) \\
\xi= & \frac{a^{2}}{2\left(a^{2}+2\right)} .
\end{aligned}
$$

In Eq. (27), $J_{\nu}(x)$ is the Bessel function of the first kind of order $\nu$. The factor $s_{m}$ appears in Esarey et al. ${ }^{10}$ It also appears in the quantity " $[J J]$ ', or ' $F_{m}(K)$ ', in the FEL/ synchrotron light literature where $K$ is the undulator/wiggler parameter. $^{34,68,78-82}$ Setting $K=a$, one finds ${ }^{78} s_{m}=\pi^{2}(1$ $\left.+K^{2} / 2\right)^{2} F_{m}(K)$. It is easy to show that $s_{m}=s_{-m}$ for all odd integers $m$. Note that the relative spectral shape of $s_{m}$ depends only on $a$ (Fig. 2), and is independent of the electron beam energy. ${ }^{12}$ The discrete spectrum for small $a$ approaches a continuum for $a^{2} \gg 1$. Note the similarity of Fig. 2 and Fig. 5.30 of Ref. 34 .

The maximum values of $s_{m}$, occurring at $m=M$ with a value $s_{M}$, are shown in Fig. 3. Note that the frequency component $\omega=M \omega_{1}$ contains the highest backscattered power. In terms of the laser frequency, the frequency component $\omega$ $=N \omega_{0}$ would contain the highest backscatter power, where $N=M \omega_{1} / \omega_{0}$. The total backscatter power, $P_{T}$ (in erg/s), per unit solid angle in the $\mathbf{n}=-z$ direction is then given by $P_{T}$ $=\Sigma p_{m}$ where the sum is taken over all odd values of $m$. The following asymptotic formulas for $s_{m}, M, s_{M}, N$, and $P_{T}$ are obtained for small and large values of $a .^{90}$

For $a<0.3$,

$$
\begin{aligned}
& s_{m} \approx 0 \quad \text { for all }|m| \neq 1, \\
& M=1, \\
& s_{M}=s_{1} \approx a^{2} \pi^{2}, \\
& N=\gamma_{0}^{2}\left(1-\beta_{z 0}\right)^{2}, \\
& P_{T} \approx 13.7 a^{2} \frac{\gamma_{0}^{6}\left(1-\beta_{z 0}\right)^{6}}{(\lambda / 1 \mu \mathrm{m})^{2}} \mathrm{erg} / \mathrm{s} .
\end{aligned}
$$




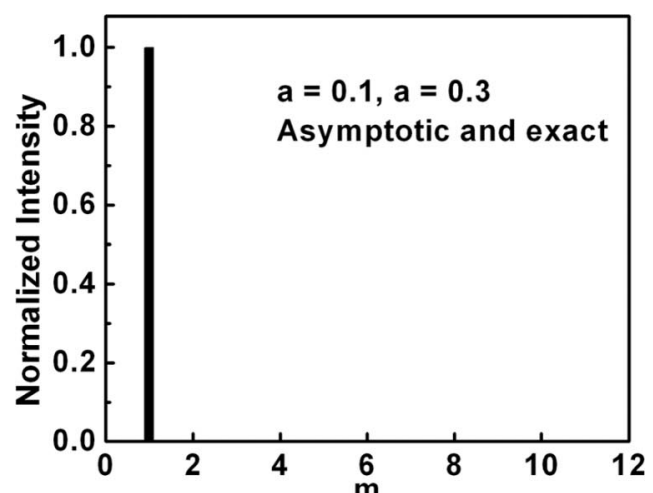

(a)

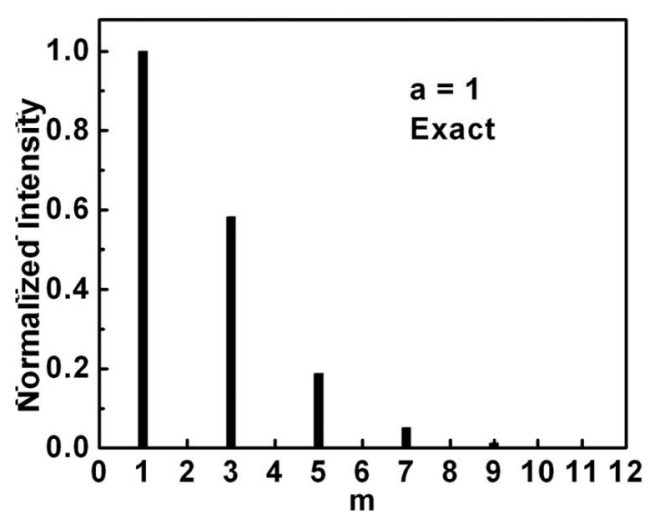

(b)
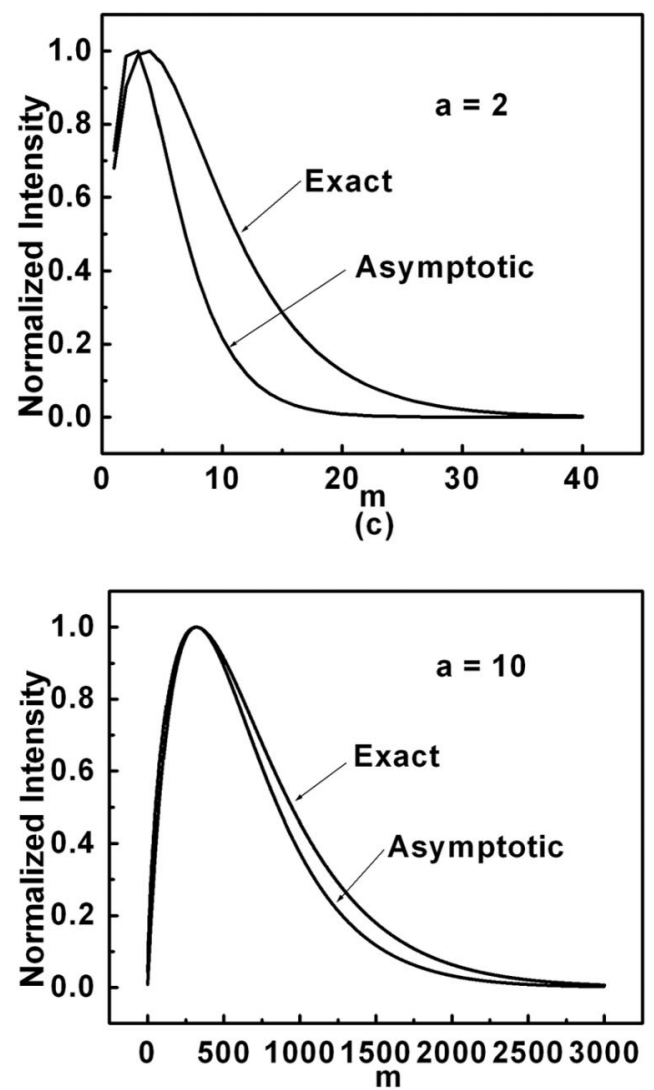

(d)

FIG. 2. Normalized spectral distribution of $s_{m}$, at frequency $\omega=m \omega_{1}$. Here, $s_{m}$ is normalized with respect to the maximum value $s_{M}$, occurring at $m=M$.
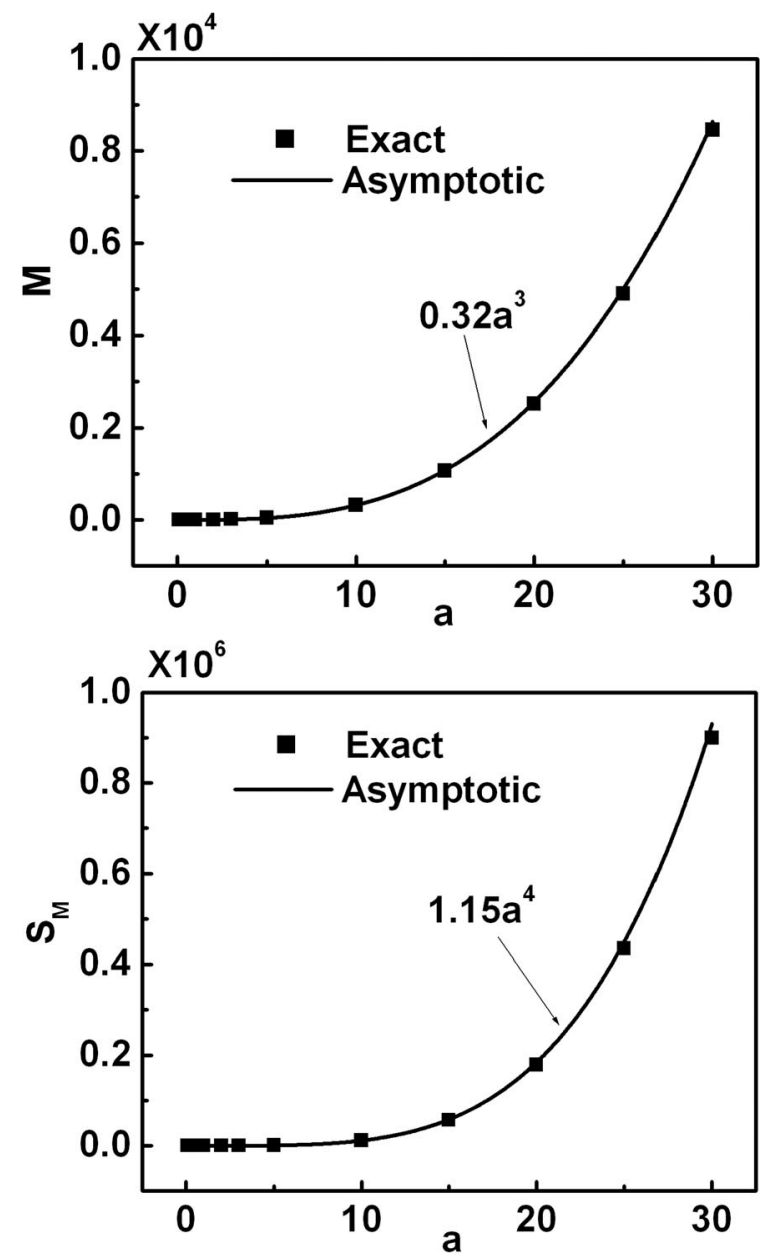

FIG. 3. Numerical values of $M$ (top) and $s_{M}$ (bottom) at various values of $a$.

For $a \gg 1$,

$$
\begin{aligned}
& s_{m} \approx s_{M}(|m| / M) e^{-(|m|-M) / M}, \\
& M=0.32 a^{3}, \\
& s_{M} \approx 1.15 a^{4}, \\
& N \approx 0.64 a \gamma_{0}^{2}\left(1-\beta_{z 0}\right)^{2}, \\
& P_{T} \approx \frac{11.1}{a} \frac{\gamma_{0}^{6}\left(1-\beta_{z 0}\right)^{6}}{(\lambda / 1 \mu \mathrm{m})^{2}} \mathrm{erg} / \mathrm{s} .
\end{aligned}
$$

Figure 2 shows the asymptotic solutions (29a) and (30a); Fig. 3 shows the asymptotic solutions (30b) and (30c). Note from Eq. (30d) that $N$ is linearly proportional to $a$, instead of $a^{3}$ for large $a$, as shown in Fig. 4 (top).

While Eq. (30e) gives only the radiated power per unit sold angle in the backscatter direction, it clearly shows that using an intense laser (large $a$ ) does not necessarily yield the brightest backscatter source. Figure 4 (bottom) suggests that an intense laser with $a=O(1)$, together with a most energetic counterpropagating electron beam, would produce the combined largest frequency upshift and the brightest back- 

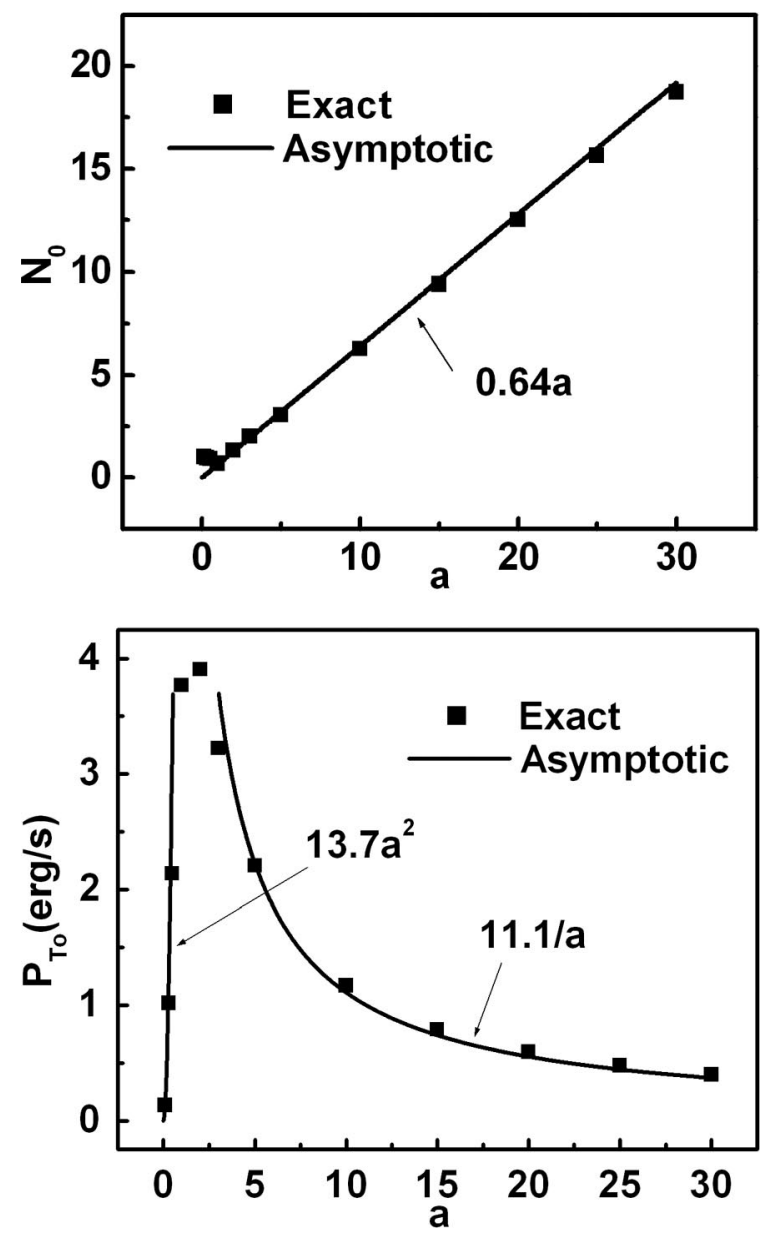

FIG. 4. (Top) The harmonic number of the laser frequency, $N_{0}$, at which maximum backscatter occurs for the special case $\beta_{z 0}=0$. For nonzero $\beta_{z 0}$, $N=\gamma_{0}^{2}\left(1-\beta_{z 0}\right)^{2} N_{0}$. (Bottom) The total backscattered power, $P_{T 0}$, per unit solid angle for the special case $\beta_{z 0}=0$, and laser wavelength $\lambda=1 \mu \mathrm{m}$. For other values of $\beta_{z 0}$ and $\lambda, P_{T}=P_{T 0} \gamma_{0}^{6}\left(1-\beta_{z 0}\right)^{6} /(\lambda / 1 \mu \mathrm{m})^{2}$.

scatter radiation..$^{90}$ The synchrotron/FEL communities have reached very similar conclusions regarding the magnetic wiggler parameter $K$ in recent years.

Note the unusual scaling of $P_{T}$, which is proportional to $\gamma_{0}^{6}$ according to Eqs. (29e) and (30e) in the highly relativistic limit $\beta_{z 0} \rightarrow-1$. To show that such a scaling is consistent with that expected from the conventional literature, consider a relativistic electron scattering head-on with a laser $\left(\beta_{z 0}\right.$ $\rightarrow-1$. See Fig. 1). The solid angle of the radiation cone is of order $\gamma^{-2},{ }^{34}$ the total power radiated by this single electron integrated over this solid angle is then of order $\gamma^{4}$. The duration of this radiation pulse is of order $(L / c) \gamma^{-2}$ if this electron is to interact only with a finite length $(L)$ of the wiggler, as can be seen by considering the (spatial) separation between the first and last photon that are produced by the electron during this electron's journey within the wiggler. Multiplying this duration and power, the total energy radiated by this electron is then of order $\gamma^{2}$, and it follows that the number of photon yield per electron is independent of $\gamma$. The last results are, of course, well-known. Use of the abovepresented argument on Eq. (29e) gives the following estimates of the number of backscatter photons by an energetic electron colliding head on with a laser of $N_{u}=L / \lambda$ optical (undulator) period:

$$
\begin{aligned}
& N_{\mathrm{ph}}(\text { per electron }) \approx \alpha a^{2} N_{u}, \\
& N_{\mathrm{ph}}(\text { total }) \approx \alpha a^{2} N_{u} N_{e} .
\end{aligned}
$$

In Eq. (31), $\alpha=e^{2} / \hbar c=1 / 137$ is the fine structure constant. Equation (31b) gives the total number of photons that are backscattered incoherently by an electron bunch that contains $N_{e}$ electrons, a well-known result in conventional synchrotron and in laser synchrotron. ${ }^{6,32,34}$ In fact, if we multiply the right-hand side of Eq. (31b) by the numerical factor $\pi / 3$, Eq. (31b) may be shown to be identical to Eq. (5.85a) on p. 183 of Attwood ${ }^{34}$ in the limit $a^{2}=K^{2} \ll 1$, and to Eq. (13) of Sprangle et al. ${ }^{6}$ We should mention that this equation of Attwood gives the total radiated power, over all frequencies, over all angles, for arbitrary value of $K=a$.

\section{REMARKS}

The above-given tutorial study is based on the simple, classical model of a single electron interacting with an infinite plane wave. While highly idealized, it suggests that the brightest x-ray source, measured as the rate of photon yield per unit solid angle per frequency, is achieved by head-on collisions of a relativistic electron beam with an intense laser with $a=O(1)$. Such a configuration, broadly known as laser synchrotron, has a striking resemblance to the conventional synchrotron/FEL both in physical terms and in the mathematical treatment. In fact, the laser parameter $a$ is found to be almost interchangeable with the FEL wiggler parameter $K=e B_{w} / m\left(k_{w} c\right) c$, where $B_{w}$ is the wiggler magnetic field and $k_{w}$ is the wiggler wavenumber. ${ }^{64,78,79,81}$ Many realistic effects that have been studied in the conventional synchrotron/FEL community ${ }^{34}$ may be immediately applied to the laser synchrotron. In the following, we discuss a few of them.

The finite length of the laser pulse contributes to a natural linewidth of the Thomson backscatter spectrum. This spectral width is proportional to $1 / N_{u}$, where $N_{u}$ is the number of the laser optical cycle. ${ }^{6,34,78-80,92}$ For small values of $N_{u}$, such as in the single digit, the spectral brightness is reduced, because it gives a much larger radiation $\operatorname{cone}^{34}$ and because the photon yield is reduced [cf. Eq. (31a)]. The spectral brightness is the rate of photon yield per unit source area per unit solid angle per unit bandwidth. The effect of the laser finite spot size requires special attention for large $a$ for Thomson scattering, because the electron's transverse excursion may become comparable to the spot size, leading to the possibility that the electron may be lost before it completes even one figure- 8 orbit. Radiation patterns including the laser's finite spot size for lower values of $a$ were given in Ref. 29.

The quality of the electron beam is the single most important factor in all short wavelength coherent radiation sources (see, e.g., Refs. 66 and 67). The number of electrons in the bunch, the electron beam emittance, the energy spread, the longitudinal and transverse dimensions of the electron bunch, all affect the brightness and the achievable pulse length of the $\mathrm{x}$-ray pulse. ${ }^{34,51,52,78,82}$ The $\mathrm{x}$-ray pulse length is 
usually limited by the length of the electron bunch, while the transverse dimension of the electron bunch needs to match that of the laser for optimal interaction. Since the spectral brightness is inversely proportional to the square of the electron beam emittance, control of beam emittance and velocity spread in a high current beam will be the decisive factor for the viability of an X-ray laser synchrotron. Likewise, the spatial coherence and temporal coherence of the laser synchrotron can be similarly addressed [cf. Chap. 8 of Ref. 34].

If the experience with the conventional synchrotron may serve as a guide, the all-optical x-ray source may consist of an intense laser with $a=O(1)$, about 100 optical cycles, and an optically produced electron bunch with comparable beam emittance and energy spread as that envisioned for the (conventional) $\mathrm{x}$-ray synchrotron (though at much lower energy, say $50 \mathrm{MeV}$, instead of $10 \mathrm{GeV}$ ). Proposals for such alloptical electron accelerators are given in Refs. 96-99.

The radiation processes studied in this paper, namely, using an electron beam in an electromagnetic wiggler, have been restricted to the spontaneous emission operated in the single particle regime, and therefore conceptually similar to the "third generation (laser) synchrotron." One might wonder whether the laser synchrotron is capable of operation in the self amplified spontaneous emission (SASE) mode, where exponential gain is projected..$^{64-77,81,92}$ The lack of mirror for $\mathrm{x}$ rays prompted the most recent developments of a SASE FEL using a high current, multi-GeV beam on a magnetic undulator in the synchrotron/FEL community, generally known as the "fourth generation synchrotron." Recent SASE experiments were reported in Refs. 77, 100, 101, and 65.

Once more, the FEL/synchrotron results may again be immediately applied to a SASE laser synchrotron source. In fact, Gover and Sprangle ${ }^{64}$ back in 1981 already gave a unified analysis on the stimulated emission for traveling wave tubes, Smith-Purcell radiator, FEL with magnetostatic or electrostatic wiggler, and FEL with an electromagnetic wiggler (the present case). All regimes, ranging from low current (low gain Compton) to high current (high gain Compton) to the space charge dominated (Raman) regimes, have been analyzed. SASE was in the high gain Compton regime, operating in what was also known as the "super-radiant" mode.

\section{ACKNOWLEDGMENTS}

We thank Sudeep Banerjee, Bahman Hafizi, and Tony Ting for many useful discussions.

This work was supported by the Office of Naval Research, the Department of Energy (Chemical Sciences, Geosciences and Biosciences Division of the Office of Basic Energy Sciences), and the National Science Foundation.

${ }^{1}$ D. Strickland and G. Mourou, Opt. Commun. 56, 216 (1985); G. Mourou and D. Umstadter, Phys. Fluids B 4, 2315 (1992); M. Perry and G. Mourou, Science 264, 917 (1994); G. Mourou and D. Umstadter, Sci. Am. 286, 80 (2002).

${ }^{2}$ R. W. Boyd, Nonlinear Optics (Academic, Boston, 1992).

${ }^{3}$ C. J. Joshi and P. B. Corkum, Phys. Today 48 (1), 36 (1995).

${ }^{4}$ F. V. Hartemann, High Field Electrodynamics (CRC Press, Boca Raton, FL, 2001).

${ }^{5}$ D. Umstadter, Phys. Plasmas 8, 1774 (2001).
${ }^{6}$ P. Sprangle, A. Ting, E. Esarey, and A. Fisher, J. Appl. Phys. 72, 5032 (1992); A. Ting, R. Fischer, A. Fisher, K. Evans, R. Burris, J. Krall, E. Esarey, and P. Sprangle, ibid. 78, 575 (1995); A. Ting, R. Fischer, A. Fisher et al., Nucl. Instrum. Methods Phys. Res. A 375, 68 (1996).

${ }^{7}$ F. V. Hartemann, Phys. Plasmas 5, 2037 (1998); Phys. Rev. E 64, 016501 (2002).

${ }^{8}$ E. S. Sarachik and G. T. Schappert, Phys. Rev. D 1, 2738 (1970).

${ }^{9}$ D. F. Alferov, Y. A. Bashmakov, and E. G. Bessonov, Sov. Phys. Tech. Phys. 18, 1336 (1974); Vachaspati, Phys. Rev. 128, 664 (1962).

${ }^{10}$ E. Esarey, S. K. Ride, and P. Sprangle, Phys. Rev. E 48, 3003 (1993).

${ }^{11}$ C. I. Castillo-Herrera and T. W. Johnston, IEEE Trans. Plasma Sci. 21, 125 (1993).

${ }^{12}$ F. V. Hartemann, Phys. Plasmas 5, 2037 (1998).

${ }^{13}$ J. E. Gunn and J. P. Ostriker, Astrophys. J. 165, 523 (1971).

${ }^{14}$ S. Y. Chen, A. Maksimchuk, and D. Umstadter, Nature (London) 396, 653 (1998).

${ }^{15}$ C. Bula, K. T. McDonald, E. J. Prebys et al., Phys. Rev. Lett. 76, 3116 (1996).

${ }^{16}$ S. Y. Chen, A. Maksimchuk, E. Esarey, and D. Umstadter, Phys. Rev. Lett. 84, 5528 (2000).

${ }^{17}$ G. Shvets, N. J. Fisch, and J.-M. Rax, Phys. Rev. Lett. 80, 2598 (1998).

${ }^{18}$ X. Liu, D. Umstadter, E. Esarey, and A. Ting, IEEE Trans. Plasma Sci. 21, 90 (1993).

${ }^{19}$ E. Esarey, A. Ting, P. Sprangle, D. Umstadter, and X. Liu, IEEE Trans. Plasma Sci. 21, 95 (1993).

${ }^{20}$ M. W. Walser, C. H. Keitel, A. Scrinzi, and T. Brabec, Phys. Rev. Lett. 85, 5082 (2000).

${ }^{21}$ P. Monot, T. Aguste, P. Gibbon et al., Phys. Rev. Lett. 74, 2953 (1995).

${ }^{22}$ S.-Y. Chen, G. S. Sarkisov, A. Maksimchuk, R. Wagner, and D. Umstadter, Phys. Rev. Lett. 80, 2610 (1998).

${ }^{23}$ A. B. Borisov, A. V. Borovskiy, V. V. Korobkin, A. M. Prokhorov, O. B. Shiryaev, X. M. Shi, T. S. Luk, A. McPherson, J. C. Solem, K. Boyer, and C. K. Rhodes, Phys. Rev. Lett. 68, 2309 (1992).

${ }^{24}$ R. Wagner, S. Y. Chen, A. Maksimchuk, and D. Umstadter, Phys. Rev. Lett. 78, 3125 (1997).

${ }^{25}$ J. S. Wurtele, Phys. Today 47 (7), 33 (1994).

${ }^{26}$ A. K. Puntajer and C. Leubner, Phys. Rev. A 40, 279 (1989).

${ }^{27}$ Y. I. Saliman and F. H. M. Faisal, Phys. Rev. A 54, 4383 (1996); 55, 3964 (1997); J. Phys. A 31, 1319 (1998).

${ }^{28}$ P. Panek, J. Z. Kaminski, and F. Ehlozky, Phys. Rev. A 65, 022712 (2002).

${ }^{29}$ U. Mohideen, H. W. K. Tom, and R. R. Freeman, J. Opt. Soc. Am. B 9, 2190 (1992).

${ }^{30}$ F. He, Y. Y. Lau, D. Umstadter, and T. Strickler, Phys. Plasmas 9, 4325 (2002); [Erratum 10, 327 (2003)].

31 "Proceedings of Workshop on Compton scattering," edited by I. V. Pogorelsky, Nuclear Instrum. Methods Phys. Res. A 455, Issue 1 (2000), and references therein.

${ }^{32}$ P. Catravas, E. Esarey, and W. P. Leemans, Meas. Sci. Technol. 12, 1 (2001)

${ }^{33}$ E. Esarey, P. Sprangle, J. Krall, and A. Ting, IEEE Trans. Plasma Sci. 24, 252 (1996).

${ }^{34} \mathrm{D}$. Attwood, Soft X-rays and Extreme Ultraviolet Radiation: Principles and Applications (Cambridge University Press, Cambridge, 1999).

${ }^{35}$ D. Attwood, K. Halbach, and K. Kim, Science 228, 1265 (1985).

${ }^{36}$ R. W. Schoenlein, H. H. W. Chong, T. E. Glover, P. A. Heimann, W. P. Leemans, H. A. Padmore, C. V. Shank, A. A. Zholents, M. S. Zolotorev, and J. S. Corlett, Acad. Sci. Paris IV, 1378 (2001).

${ }^{37}$ R. W. Schoenlein, W. P. Leemans, A. H. Chin, P. Volfbeyn, T. E. Glover, P. Balling, M. Zolotorev, K.-J. Kim, S. Chattopadhyay, and C. V. Shank, Science 274, 236 (1996).

${ }^{38}$ B. G. Englert and E. A. Rinehart, Phys. Rev. A 28, 1539 (1983).

${ }^{39}$ D. D. Meyerhofer, J. P. Knauer, S. J. McNaught, and C. I. Moore, J. Opt. Soc. Am. B 13, 113 (1996).

${ }^{40}$ S.-Y. Chen, A. Maksimchuk, and D. Umstadter, Nature (London) 396, 653 (1998).

${ }^{41}$ C. Bamber, S. J. Boege, T. Koffas et al., Phys. Rev. D 60, 092004 (1999).

${ }^{42}$ S. Y. Chen, A. Maksimchuk, E. Esarey, and D. Umstadter, Phys. Rev. Lett. 84, 5528 (2000).

${ }^{43}$ I. Christov, M. M. Murnane, and H. C. Kepteyn, Opt. Commun. 148, 75 (1998).

${ }^{44}$ I. Yu. Kostyukov, Phys. Rev. E 59, 1122 (1999).

${ }^{45}$ A. E. Kaplan and P. L. Shkolnikov, Phys. Rev. Lett. 88, 074801 (2002); 89, 199502 (2002); G. Stupakov and M. Zolotorev, ibid. 89, 199501 (2002). 
${ }^{46}$ F. V. Hartemann, Phys. Plasmas 5, 2037 (1998); Phys. Rev. E 64, 016501 (2002).

${ }^{47}$ K. Matsukado, I. Endo, T. Takahashi, M. Iinuma, S. Sakabe, F. Sato, Y. Izawa, and T. Tauchi, Nucl. Instrum. Methods Phys. Res. A 455, 46 (2000).

${ }^{48}$ H. Kotaki, M. Kando, H. Dewa, S. Kondo, T. Watanabe, T. Ueda, K. Kinoshita, K. Yoshii, M. Uesaka, and K. Nakajima, Nucl. Instrum. Methods Phys. Res. A 455, 166 (2000).

${ }^{49}$ S. Kashiwagi, M. Washio, T. Kobuki et al., Nucl. Instrum. Methods Phys. Res. A 455, 36 (2000).

${ }^{50}$ V. Malka, J. Faure, J. R. Marques et al., Phys. Plasmas 8, 2605 (2001).

${ }^{51}$ K. J. Kim, S. Chattopadhyay, and C. V. Shank, Nucl. Instrum. Methods Phys. Res. A 341, 351 (1994)

${ }^{52}$ W. P. Leemans, R. W. Schoenlein, P. Volfbeyn, A. H. Chin, T. E. Glover, P. Balling, M. Zolotorev, K. J. Kim, S. Chattopadhyay, and C. V. Shank, Phys. Rev. Lett. 77, 4182 (1996).

${ }^{53}$ V. N. Litvinenko, B. Burnham, M. Emamian et al., Phys. Rev. Lett. 78, 4569 (1997).

${ }^{54}$ I. Ben-Zvi, M. Babzien, E. B. Blum, Proceedings of the 2001 Particle Accelerator Conference, Chicago, edited by P. Lucas (IEEE, Piscataway, NJ, 2001), p. 350.

${ }^{55}$ I. V. Bazarov, D. H. Bilderback, S. M. Gruner, H. S. Padamsee, R. Talman, M. Tigner, G. A. Kraft, L. Merminga, and C. K. Sinclair, Proceedings of the Particle Accelerator Conference (IEEE, Piscataway, NJ, 2001), p. 230.

${ }^{56} \mathrm{R}$. Tatchyn, J. Arthur, M. Baltay et al., Nucl. Instrum. Methods Phys. Res. A 375, 274 (1996).

${ }^{57}$ W. P. Leemans, R. W. Schoenlein, P. Volfbeyn, A. H. Chin, T. E. Glover, P. Balling, M. Zolotorev, K.-J. Kim, S. Chattopadhyay, and C. V. Shank, IEEE J. Quantum Electron. 33, 1925 (1997).

${ }^{58}$ W. Schoenlein, S. Chattopadhyay, H. H. W. Chong, T. E. Glover, P. A. Heimann, W. P. Leemans, C. V. Shank, A. Zholents, and M. Zolotorev, Science 287, 2237 (2000).

${ }^{59}$ J. Corlett, S. DeSantis, N. Hartman et al., in Ref. 54, p. 2635.

${ }^{60}$ I. V. Pogorelsky, I. Ben-Zvi, X. J. Wang, and T. Hirose, Nucl. Instrum. Methods Phys. Res. A 455, 176 (2000).

${ }^{61}$ I. V. Pogorelsky, Nucl. Instrum. Methods Phys. Res. A 411, 172 (1998)

${ }^{62}$ Y. Ueshima, Y. Kishimoto, A. Sasaki, and T. Tajima, Laser Part. Beams 17, 45 (1999).

${ }^{63}$ J. Yang, M. Washio, A. Endo, and T. Hori, Nucl. Instrum. Methods Phys. Res. A 428, 556 (1999).

${ }^{64}$ A. Gover and P. Sprangle, IEEE J. Quantum Electron QE17, 1196 (1981); P. Sprangle, C. M. Tang, and C. W. Roberson, Nucl. Instrum. Methods Phys. Res. A 239, 1 (1985); P. Sprangle and A. T. Drobot, J. Appl. Phys. 50, 2652 (1979).

${ }^{65}$ A. Bakhtyai, J. E. Walsh, and J. H. Brownell, Phys. Rev. E 65, 066503 (2002).

${ }^{66} \mathrm{~L}$. Schachter, Beam-Wave Interaction in Periodic and Quasi-Periodic Structures (Springer, Berlin, 1996).

${ }^{67}$ C. W. Roberson, IEEE J. Quantum Electron. 21, 860 (1985); C. W. Roberson and P. Sprangle, Phys. Fluids B 1, 3 (1989).

${ }^{68}$ W. Colson, IEEE J. Quantum Electron. 17, 1417 (1981).

${ }^{69}$ C. Pellegrini, Nucl. Instrum. Methods Phys. Res. A 272, 364 (1988).

${ }^{70}$ W. B. Colson and C. Blau, Nucl. Instrum. Methods Phys. Res. A 272, 386 (1988).

${ }^{71}$ A. Gover and Z. Livni, Opt. Commun. 26, 375 (1978).

${ }^{72}$ R. Bonifacio, C. Pellegrini, and L. M. Narducci, Opt. Commun. 50, 373 (1984).
${ }^{73}$ A. Yariv and C. Shih, Opt. Commun. 24, 233 (1978).

${ }^{74}$ M. Xie and D. A. G. Deacon, Nucl. Instrum. Methods Phys. Res. A 250, 426 (1986).

${ }^{75}$ K. Kim, Nucl. Instrum. Methods Phys. Res. A 250, 396 (1986).

${ }^{76}$ K. Kim, Phys. Rev. Lett. 57, 1871 (1986).

${ }^{77}$ M. Babzien, I. Ben-Zvi, P. Catravas, J.-M. Fang, T. C. Marshall, X. J. Wang, J. S. Wurtele, V. Yakimenko, and L. H. Yu, Phys. Rev. E 57, 6093 (1998).

${ }^{78}$ K. J. Kim, American Institute of Physics Conference Proceedings No. 184, edited by M. Month and M. Dienes (AIP, New York, 1989), p. 565; Nucl. Instrum. Methods Phys. Res. A 250, 396 (1986); Optical Engineering (Bellingham, Washington, 1995), Vol. 34, p. 342.

${ }^{79}$ S. Krinsky, M. L. Perlman, and R. E. Watson, in Handbook on Synchrotron Radiation, edited by E. E. Koch (North-Holland, Amsterdam, 1983), Vol. 1A, Chap. 2, p. 65.

${ }^{80}$ C. A. Brau, Free-Electron Lasers (Academic, San Diego, 1990).

${ }^{81}$ P. G. O'Shea and J. B. Murphy, "Free electron lasers and synchrotron light sources," in Handbook of Lasers (Institute of Physics, Bristol, 2003, to be published).

${ }^{82}$ K. J. Kim, Nucl. Instrum. Methods Phys. Res. A 246, 67 (1986); 246, 71 (1986).

${ }^{83}$ P. G. O'Shea and H. P. Freund, Science 292, 1853 (2001); W. B. Colson, E. D. Johnson, M. J. Kelly, and H. A. Swettman, Phys. Today 55 (1), 35 (2002).

${ }^{84}$ P. Panek, J. Z. Kaminski, and F. Ehlotzky, Phys. Rev. A 65, 022712 (2002).

${ }^{85}$ P. Gibson, IEEE J. Quantum Electron. 33, 1915 (1997).

${ }^{86}$ Q. Park, R. W. Boyd, J. E. Sipe, and A. L. Gaeta, IEEE J. Quantum Electron. 8, 413 (2002).

${ }^{87}$ F. V. Hartemann, Phys. Plasmas 5, 2037 (1998).

${ }^{88}$ F. V. Hartemann and A. K. Kerman, Phys. Rev. Lett. 76, 624 (1996).

${ }^{89}$ F. V. Hartemann, H. A. Baldis, A. K. Kerman, A. Le Foll, N. C. Luhmann, Jr., and B. Rupp, Phys. Rev. E 64, 016501 (2001).

${ }^{90}$ F. He, Y. Y. Lau, D. Umstadter, and R. Kowalczyk, Phys. Rev. Lett. 90, 055002 (2003).

${ }^{91}$ J. D. Jackson, Classical Electrodynamics (Wiley, New York, 1962), p. 480.

${ }^{92}$ H. P. Freund, P. Sprangle, D. Dillenburg, E. H. da Jornada, B. Liberman, and R. S. Schneider, Phys. Rev. A 24, 1965 (1981); H. P. Freund, T. M. Antonsen, Jr., Principles of Free-Electron Lasers (Chapman and Hall, London, 1992).

${ }^{93}$ W. H. Press, S. A. Teukolsky, W. T. Vetterling, and B. P. Flannery, Numerical Recipe in Fortran 77, 2nd ed. (Cambridge University Press, Cambridge, 1996), Chap. 4.

${ }^{94}$ P. B. Corkum, Phys. Rev. Lett. 71, 1994 (1993).

${ }^{95}$ S. Banerjee, A. R. Valenzuela, R. C. Shah, A. Maksimchuk, and D. Umstadter, J. Opt. Soc. Am. B 20, 182 (2003).

${ }^{96}$ D. Umstadter, J. K. Kim, and E. Dodd, Phys. Rev. Lett. 76, 2073 (1996).

${ }^{97}$ E. Esarey, R. F. Hubbard, W. P. Leemans, A. Ting, and P. Sprangle, Phys. Rev. Lett. 79, 2682 (1997).

${ }^{98}$ E. Esarey, C. B. Schroeder, W. P. Leemans, and B. Hafizi, Phys. Plasmas 6, 2262 (1999).

${ }^{99}$ R. G. Hemker, K.-C. Tzeng, W. B. Mori, C. E. Clayton, and T. Katsouleas, Phys. Rev. E 57, 5920 (1998).

${ }^{100}$ J. Andruszkow, B. Aune, V. Ayvazyan et al., Phys. Rev. Lett. 85, 3825 (2000).

${ }^{101}$ S. V. Milton, E. Gluskin, N. D. Arnold et al., Science 292, 2037 (2001). 